\title{
Solvent Extraction of Scandium and Yttrium Using Carboxylic Acid
}

\author{
Jeong-Yi Moon, Syouhei NishiHAMA, and Kazuharu YoshizUKA* \\ Department of Chemical Engineering, The University of Kitakyushu, Hibikino 1-1, Kitakyushu 808-0135, Japan
}

\begin{abstract}
Separation of scandium (Sc) from yttrium $(\mathrm{Y})$ in aqueous chloride media by solvent extraction using Versatic acid 10 was investigated. Conventional slope analysis method revealed the extraction equilibrium formulation of the metals. Sc is more strongly extracted than $Y$ by Versatic acid 10, and the separation factor of the metals is quite high at $1.93 \times 10^{4}$. Complete stripping of Sc from the loaded organic solution of Versatic acid 10 was achieved by $3 \mathrm{M} \mathrm{HCl}$ solution. Then Versatic acid 10 was applied to solvent impregnated resin (SIR), separation of Sc was progressed via Langmuir adsorption model.
\end{abstract}

\section{Introduction}

Scandium ( $\mathrm{Sc}$ ) is one of the rare earth metals in high demand, providing excellent characteristics for various industrial applications (USGS, 2018; Paulick and Machacek, 2017). Although Sc is not particularly rare in the overall crustal abundance, there is little reserves of scandium-rich minerals. Since Sc is similar to yttrium (Y) and the heavy lanthanides owing to its small ionic radius, it is commonly found together with $\mathrm{Y}$ and heavy lanthanides. Thus, from the perspective of resource sustainability, the development of high-efficiency separation processes has become very important in most countries such as Japan, which has the second largest scandium in the world, relies on imports from China for most of its demand. Thus, separation of Sc from yttrium (Y) and other rare earths is required.

Separation of rare earth metals is generally performed hydrometallurgical processes. Solvent extraction is one of the most widely used hydrometallurgical processes for the separation and purification of rare earth elements (Wang and Li, 1995 ; Wang et al., 2002; Jia et al., 2009). Solvent extraction is carried out using functional groups of particular interest, taking into account such disadvantages as use of large amounts of organic solvents with the advantages of high selectivity and ease of scale-up. Therefore, solvent impregnated resin (SIR) is getting attention which is environmentally friendly, possessing high selectivity by integrating the unique features and process advantages of solvent extraction and ion exchange. The major disadvantage of SIRs is loss of extractant due to solubility in the aqueous phase, which can be minimized by coating with polymers and chemical crosslinking (Trochimzuk et al., 2004).

When considering the separation of rare earth elements, organophosphorus compounds such as bis (2- ethylhexyl)phosphoric acid (D2EHPA) (OchsenkuhnPetropupu et al., 1995) or 2-ethylhexyl phosphonic acid mono-2-ethylhexyl ester (PC-88A) (Lee et al., 2005) are generally used. Although Sc could be extracted separately from other rare earths, it is difficult to strip the rare earth elements from the loaded organic solution due to high extractability. Thus, in this work, carboxylic acid, such as naphtehenic acid and Versatic acid (Zheng et al., 1991), which are widely used for extracting scandium, is applied. When naphthenic acid in kerosene was used as the organic phase and hydrochloric acid was used as the stripping solution, the separation factor of Sc over all rare earth elements was very high and the order of separation of Sc was far ahead of Y (Liao et al., 2001). Although $\mathrm{Sc}^{3+}$ was extracted separately at $\mathrm{pH} 3$ to 5 from other rare earth metal ions, extraction mechanism by Versatic acid 10 was not demonstrated (Baba et al., 2014).

In the present study, therefore, separation and recovery of Sc and Y using Vesatic acid 10 have been investigated. The extraction and stripping behavior of solvent extraction were first investigated in a conventional extraction system. Conventional slope analysis method revealed the extraction equilibrium formulation of the metals. Versatic acid 10 impregnated SIR was finally employed in batchwise adsorption.

\section{Experimental}

\subsection{Reagents}

Versatic acid 10 was supplied by HEXION Specialty Chemicals Co., Ltd (Tokyo, Japan), scandium oxide and yttrium oxide were supplied by Nippon Yttrium Co., Inc. (Fukuoka, Japan). IP Solvent 2835, a synthetic isoparaffinic hydrocarbon having 99.8 vol\% or more isoparaffin was supplied by Idemitsu Kosan Co., Ltd (Tokyo,

\footnotetext{
* Corresponding author: yoshizuka@kitakyu-u.ac.jp
} 
Japan). Diaion HP2MG, a methacrylic ester copolymer containing no functional group, was supplied by Nippon Rensui Co., Ltd. (Tokyo, Japan). All other organic and inorganic reagents were purchased from Wako Pure Chemical Industries, Ltd. (Osaka, Japan) as analyticalgrade reagents.

\subsection{Extraction of Sc}

Aqueous rare earth chloride solutions were prepared by dissolving each oxide in 1 or $2 \mathrm{~mol} / \mathrm{L} \mathrm{HCl}$ solution, and then diluted with deionized water to obtain $1 \mathrm{mmol} / \mathrm{L} \mathrm{Sc}$ or $\mathrm{Y}$ aqueous solutions. The $\mathrm{pH}$ of the aqueous solution was adjusted using $\mathrm{HCl}$ or $\mathrm{NaOH}$. Organic solutions were prepared by diluting Versatic acid 10 in IP Solvent 2835. Concentrations of the extractant as a dimeric species base are shown in the figure captions. Extraction of Sc and Y was carried out by shaking the organic and aqueous solutions at a volume ratio of $1: 1$ at $298 \mathrm{~K}$ for more than $6 \mathrm{~h}$. Concentrations of the metals in the resultant aqueous solutions were determined using an inductively coupled plasma atomic emission spectrometer (ICP-AES, Shimadzu ICPE-9000) and those in the organic solutions were calculated based on material balance. The $\mathrm{pH}$ was measured by a $\mathrm{pH}$ meter (Horiba F-74). The distribution ratio $(D)$ was defined as:

$$
D=[\overline{\mathrm{M}}] /[\mathrm{M}]
$$

where $[\overline{\mathrm{M}}]$ and $[\mathrm{M}]$ are concentrations of metals in the organic phase and aqueous phase, respectively.

Stripping experiments were carried out using the metal loaded organic solution. In this case, the extraction was carried out in the same manner as described above, and the metal loaded organic solution was used in the stripping experiments. Stripping was performed by contacting the loaded organic solution with different concentrations of $\mathrm{HCl}(2-3 \mathrm{~mol} / \mathrm{L})$ at a volume ratio $=$ $1: 1$, and the mixture was shaken at $298 \mathrm{~K}$ for more than $3 \mathrm{~h}$. The concentrations of the metals in the resultant aqueous solutions were determined by ICP-AES and those in the organic solutions were calculated based on material balance.

\subsection{Preparation and coating of SIR}

The SIR was prepared by the following method (Kabay et al., 2010; Nishihama et al., 2013). HP2MG was washed with methanol and dried in vacuo. Washed HP2MG (2.5 g) was then immersed overnight in a 0.2 $\mathrm{mol} / \mathrm{L}$ Versatic acid 10 -toluene solution $(50 \mathrm{~mL})$. The toluene was then removed by evaporation, and the resin dried in vacuo for $24 \mathrm{~h}$. The amount of Versatic acid 10 impregnated in the resin was $8.23 \mathrm{mmol} / \mathrm{g}$. Coating of SIRs were conducted using PVA, of polymerization degree ca. 2000, crosslinked by glutaraldehyde (GA). The prepared SIR ( $3 \mathrm{~g})$ was immersed in an aqueous solution $(50 \mathrm{~mL}$ ) containing $3 \mathrm{wt} \%$ PVA and $1 \mathrm{wt} \%$ acetamide and the mixture was shaken for $17 \mathrm{~h}$. The resultant suspension was then mixed with $1 \mathrm{~mol} / \mathrm{L} \mathrm{KCl}$ aqueous solution (10 $\mathrm{mL}$ ) and the mixture was shaken for $24 \mathrm{~h}$. The pre-treated SIR was filtered and resuspended in a $0.32 \mathrm{~mol} / \mathrm{L} \mathrm{H}_{2} \mathrm{SO}_{4}$ solution $(10 \mathrm{~mL})$ for $1 \mathrm{~h} .0 .32 \mathrm{~mol} / \mathrm{L} \mathrm{GA}$ aqueous solution
$(10 \mathrm{~mL})$ was then added, followed by shaking the mixture for $24 \mathrm{~h}$, to complete crosslinking. The coated SIR obtained was filtered, washed with deionized water, and then dried. Hereafter, SIR used in the present study was all coated SIR.

\subsection{Adsorption of Sc with SIR}

Aqueous rare earth chloride solutions were prepared by dissolving each oxide in 1 or $2 \mathrm{~mol} / \mathrm{L} \mathrm{HCl}$ solution, and then diluted with deionized water to obtain $1 \mathrm{mmol} / \mathrm{L} \mathrm{Sc}$ and $\mathrm{Y}$ aqueous solutions. The $\mathrm{pH}$ of the aqueous solution was adjusted using $\mathrm{HCl}$ or $\mathrm{NaOH}$. The SIR $(20 \mathrm{mg})$ was added to $20 \mathrm{~mL}$ of aqueous solution, and the suspended mixture was shaken at $298 \mathrm{~K}$ for $24 \mathrm{~h}$. The concentrations of the metal ions were determined, after filtration of the resin, with an ICP-AES. The amount of adsorbed metal ion, $q(\mathrm{mmol} / \mathrm{g})$, is defined as:

$$
q=\left([\mathrm{M}]_{\mathrm{ini}}-[\mathrm{M}]_{\mathrm{eq}}\right) \times V / W
$$

where $[\mathrm{M}]_{\mathrm{ini}}$ and $[\mathrm{M}]_{\mathrm{eq}}$ are initial and equilibrium concentrations of metals in the solution $(\mathrm{mmol} / \mathrm{L})$, respectively, $V$ is the volume of the solution $(\mathrm{L})$, and $W$ is the weight of the resin $(\mathrm{g})$.

Elution experiments were carried out using the metal loaded SIR. In this case, the adsorption was carried out in the same manner as described above, and the metal loaded SIR was used. Elution was performed by adding $10 \mathrm{mg}$ of metal adsorbed SIR to $10 \mathrm{~mL}$ of different concentrations of $\mathrm{HCl}(1-3 \mathrm{~mol} / \mathrm{L})$, and the mixture was shaken at $298 \mathrm{~K}$ for more than $24 \mathrm{~h}$. The concentrations of the metal ions were determined with ICP-AES. The elution rate was calculated by material balance.

\section{Results and Discussion}

\subsection{Extraction of Sc}

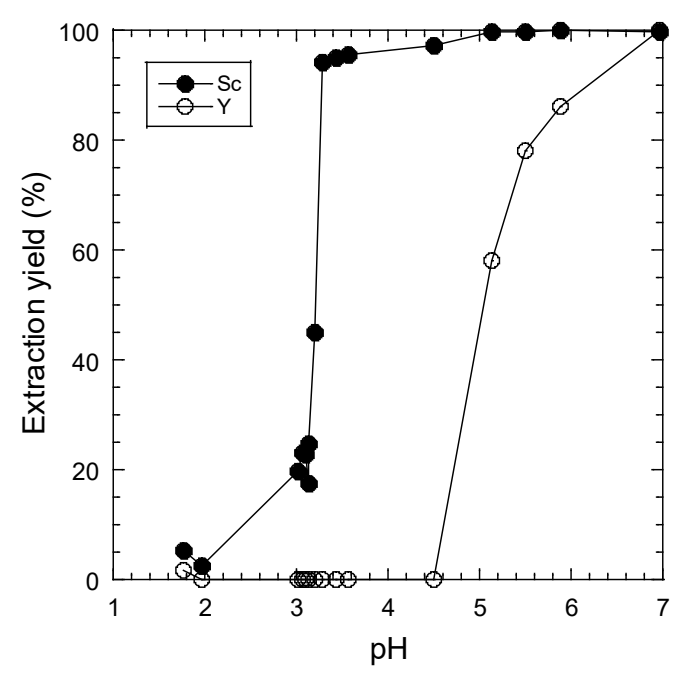

Figure 1. Effect of $\mathrm{pH}$ on the extraction yield

Figure 1 shows the effect of $\mathrm{pH}$ on the extraction yield of Sc and Y. Extraction of Sc proceeds at $\mathrm{pH} 3-4$, while extraction of $\mathrm{Y}$ proceeds at $\mathrm{pH}>4.5$. Thus, separation of $\mathrm{Sc}$ from $\mathrm{Y}$ is expected to be acheived at $\mathrm{pH} 3-4$. 


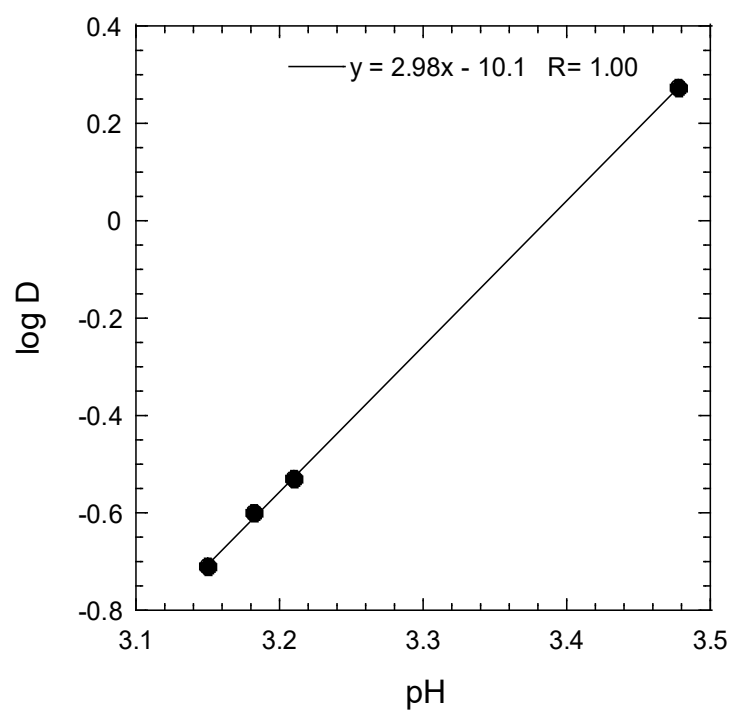

Figure 2. Effect of $\mathrm{pH}$ on the distribution ratios of Sc. $\left[\overline{(\mathrm{RH})_{2}}\right]_{\text {feed }}=0.0354 \mathrm{~mol} / \mathrm{L},[\mathrm{Sc}]_{\text {feed }}=1.00$ $\mathrm{mmol} / \mathrm{L}$

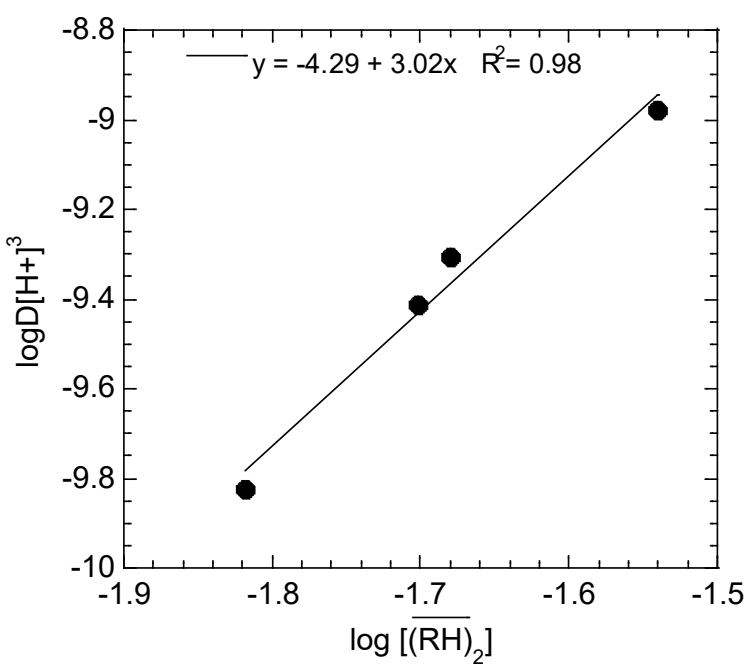

Figure 3. Effect of concentration of dimeric extractants on the normalized distribution ratios of $\mathrm{Sc}$. $\left[\overline{(\mathrm{RH})_{2}}\right]_{\text {feed }}=0.0354 \mathrm{~mol} / \mathrm{L},[\mathrm{Sc}]$ feed $=1.00$ $\mathrm{mmol} / \mathrm{L}$

Figure 2 shows the effect of $\mathrm{pH}$ on the distribution ratio of Sc from $\mathrm{pH} 3.1$ to 3.5 where extraction of Y hardly occurs. For Sc, a linear relationship with a slope of 3 between $\mathrm{pH}$ and $\log D$ was obtained, indicating that the extraction proceeds by cation exchange between scandium and the three protons. Figure 3 shows the effect of the concentration of the dimerized extractant on the distribution ratio. The relationship between $\log D\left[\mathrm{H}^{+}\right]^{3}$ and $\left.\log \left[(\mathrm{RH})_{2}\right)\right]_{\text {feed }}$ for Sc showed a dependency of slope 3 , so that three dimeric extractants participate in the extraction of each metal ion. Based on the results of the slope analysis, the extraction equilibrium for $\mathrm{Sc}$ is formulated as equation (3).

$$
\mathrm{Sc}^{3+}+3 \overline{(\mathrm{RH})_{2}} \leftrightarrow \overline{\mathrm{ScR}_{3}(\mathrm{RH})_{3}}+3 \mathrm{H}^{+}
$$

$K_{\text {ex }}$ value for Sc is $1.93 \times 10^{4}$ which is quite high, and it implies the stripping of Sc is expected to be difficult.

Stripping of the loaded Sc was then investigated. Table 1 lists the stripping yields of Sc from the loaded organic solutions containing Versatic acid 10 with different concentrations of $\mathrm{HCl}$. The stripping yield of Sc reached $100 \%$ by $3 \mathrm{~mol} / \mathrm{L} \mathrm{HCl}$.

Table 1. Stripping rate of Sc using different concentrations of hydrochloric acid

\begin{tabular}{cc}
\hline $\mathbf{H C l}(\mathbf{m o l} / \mathbf{L})$ & Stripping rate (\%) \\
\hline 1 & 49.2 \\
2 & 87.7 \\
3 & 100 \\
\hline
\end{tabular}

\subsection{Adsorption of Sc and $\mathrm{Y}$ with SIR}

Figure 4 shows the effect of $\mathrm{pH}$ on the adsorption of Sc and $\mathrm{Y}$ with Versatic acid 10 SIR in the binary solution. From $\mathrm{pH} 1.5$ to $3.5 \mathrm{Sc}$ and $\mathrm{Y}$ are adsorbed and $\mathrm{Sc}$ was maximum at $\mathrm{pH} 2.5$, while $\mathrm{Y}$ was also maximum at $\mathrm{pH}$ 2.7 .

Adsorption isotherm of Sc in $\mathrm{pH} 2.8$ with Versatic acid 10 is shown in Figure 5. Assuming that the adsorption is of Langmuir mechanism, as shown in equation (4),

$$
q=\frac{q_{0} K[\mathrm{M}]}{1+K[\mathrm{M}]}
$$

where $q_{0}$ is the maximum adsorption amount $(\mathrm{mmol} / \mathrm{g})$ and $K$ is the adsorption equilibrium constant $(\mathrm{L} / \mathrm{mmol})$. From the linear relationship of the Langmuir mechanism, Figure 5 (b), the adsorption of Sc with the Versatic acid $10 \mathrm{SIR}$ is of Langmuir mechanism. The maximum adsorption amounts of Sc calculated was $1.31 \mathrm{mmol} / \mathrm{g}$, and the adsorption equilibrium constant was 0.664 $\mathrm{L} / \mathrm{mmol}$, respectively.

Elution of adsorbed Sc was then investigated. Table 2 lists the elution yields of Sc from the adsorbed resin with different concentrations of $\mathrm{HCl}$. The stripping yield of Sc reached only $52.2 \%$ by $3 \mathrm{M} \mathrm{HCl}$.

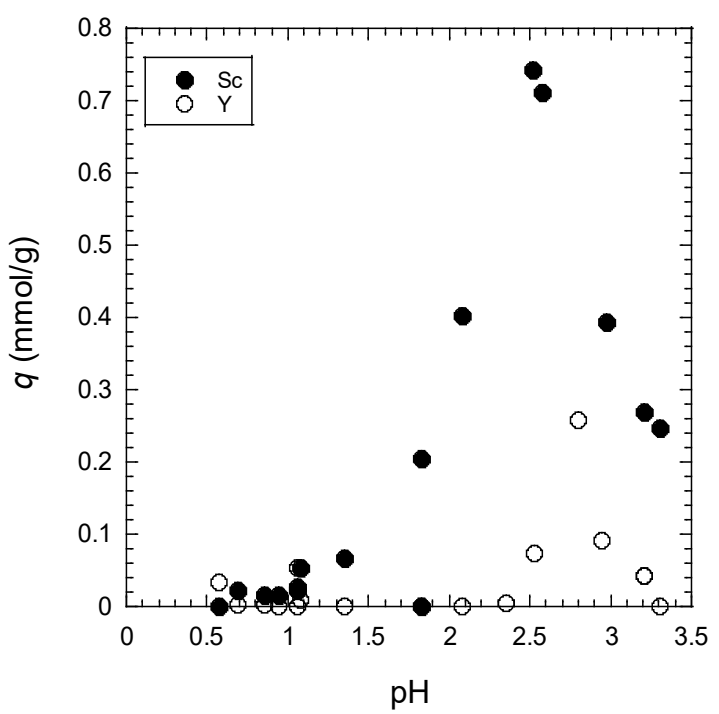

Figure 4. Effect of $\mathrm{pH}$ on amount of adsorption of Sc and $\mathrm{Y}$ with Versatic acid 10 SIR 
Table 2. Elution yield of Sc using different concentrations of hydrochloric acid

\begin{tabular}{cc}
\hline $\mathbf{H C l}(\mathbf{m o l} / \mathbf{L})$ & Stripping rate $(\%)$ \\
\hline 1 & 25.4 \\
2 & 31.0 \\
3 & 52.2 \\
\hline
\end{tabular}
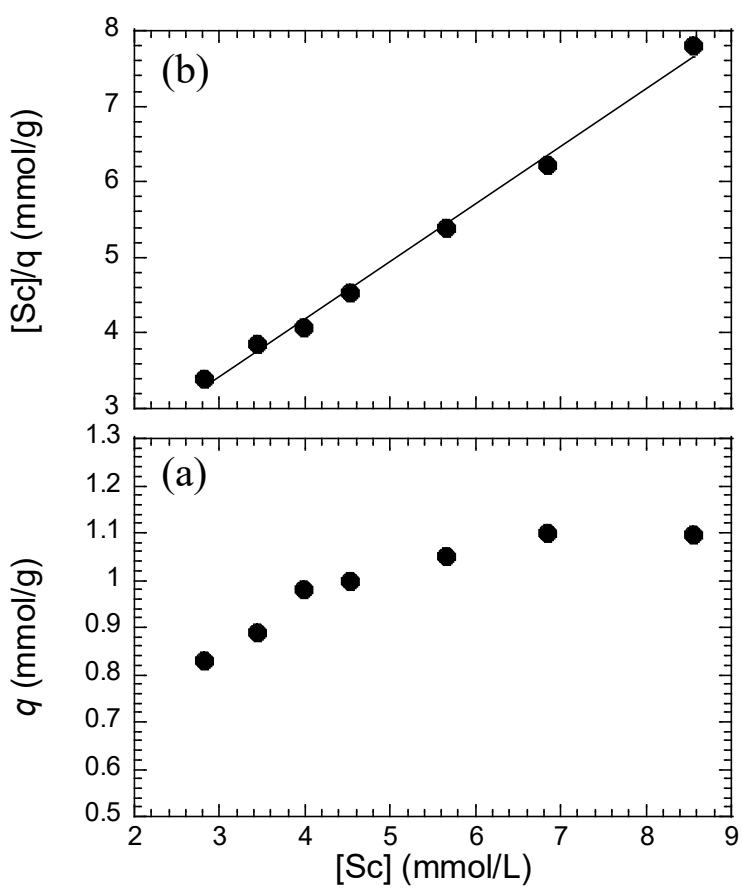

Figure 5. Adsorption isotherm and linearized relationship Langmuir mechanism of Sc with Versatic acid 10 SIR. $y=0.760 x+1.15, \mathrm{r}^{2}=0.99$

\section{Conclusion}

Extraction and stripping of Sc with Versatic acid 10 and adsorption using Versatic acid 10 SIR were investigated with the following results.

1. The extraction of Sc was proceeded by the conventional cation exchange mechanism and equilibrium constant was quite high at $1.93 \times 10^{4}$.

2. The stripping yield of $\mathrm{Sc}$ from Versatic acid 10 reached $100 \%$ using $3.0 \mathrm{~mol} / \mathrm{L} \mathrm{HCl}$ solution as the stripping reagent.

3. Versatic acid 10 SIR can adsorbs $\mathrm{Sc}$ under specific $\mathrm{pH}$ region. Adsorption of Sc by Versatic acid 10 follows the Langmuir adsorption model and the maximum adsorption amounts of $\mathrm{Pt}$ was $1.31 \mathrm{mmol} / \mathrm{g}$, and the adsorption equilibrium constant $\mathrm{K}$ was $0.664 \mathrm{~L} / \mathrm{mmol}$, respectively.

\section{References}

Baba. Y, A.Fukami, F. Kubota, N. Kamiya, and M. Goto; "Selective Extraction of Scandium from Yttrium and Lanthanides with Amic Acid-Type Extractant Containing Alkylamide and Glycine Moieties," RSC
Adv., 4, 50726-50730 (2014)

Jia, Q., S. Tong, Z. Li, W. Zhou, H. Li, and S. Meng; "Solvent Extraction of Rare Earth Elements with Mixtures of Sec-Octylphenoxy Acetic Acid and bis(2,4,4-trimethylpentyl) Dithiophosphinic Acid," Sep. Purif. Technol., 64, 345-350 (2009)

Kabay. N, J. Cortina, A. Trochimczuk, and S. Streat; "Solvent-Impregnated Resins (SIRs) - Methods of Preparation and Their Applications," React. Funct. Polym., 70, 484-496 (2010)

Lee. M. S., J. Y. Lee, J. S. Kim, and G. S. Lee; "Solvent Extraction of Neodymium Ions from Hydrochloric Acid Solution Using PC88A and Saponified PC88A," Sep. Purif. Technol., 46, 72-78 (2005)

Liao. C., J. Jia, G. Xu, C. Yan, B. Li, and G. Xu; "Extraction of Scandium from Ion-Adsorptive Rare Earth Deposit by Naphthenic Acid," Alloys. Compd., 323-324, 833-837 (2001)

Nishihama. S, K. Kohata, and K. Yoshizuka; "Separation of Lanthanum and Cerium Using a Coated Solvent Impregnated Resin," Sep. Purif. Technol., 118, 511-518 (2013)

Ochsenkuhn-Petropupu, M., T. Lyberopulu, and G. Parissakis, "Selective Separation and Determination of Scandium from Yttrium and Lanthanides in Red Mud by a Combined ion Exchange/Solvent Extraction Method," Anal. Chem. Acta, 315, 231-237 (1995)

Paulick, H. and E. Machacek ; "The Global Rare Earth Element Exploration Boom: An Analysis of Resources Outside of China and Discussion of Development Perspectives," Resources Policy, 52, 134-153 (2017)

Trochimczuk, A,. N. Kabay, M. Arda, and M. Streat; "Stabilization of Solvent Impregnated Resins (SIRs) by Coating with Water Soluble Polymers and Chemical Crosslinking," React. Funct. Polym., 59, 1-7 (2004)

USGS ; Mineral Commodity Summaries 2018, pp. 132-133, U.S. Geological Survey, Reston, USA (2018)

Wang, C. and D. Q. Li, "Solvent Extraction of Sc(III), $\mathrm{Zr}(\mathrm{IV}), \quad \mathrm{Th}(\mathrm{IV}), \quad \mathrm{Fe}(\mathrm{III}), \quad$ and Lu(III) with Thiosubstituted Organophosphinic Acid Extractants," Solvent Extr. Ion Exch., 13, 503-523 (1995)

Wang. Y. G., S. T. Yue, D. Q. Li, M. J. Jin, and C. Z. Li; "Solvent Extraction of Scandium(III), Yttrium(III), Lanthanides(III), and Divalent Metal Ions with SecNonylphenoxylacetic Acid," Solvent Extr. Ion Exch., 20, 701-716 (2002)

Zheng, D., N. B. Gray, and G. W. Stevens; 
"Comparison of Naphtenic Acid, Versatic Acid and D2EHPA for the Separation of Rare Earths," Solvent Extr. Ion Exch., 1, 85-102 (1991) 